\title{
PRONTIDÃO À TECNOLOGIA: UM ESTUDO SOBRE A APLICAÇÃO DA TECHNOLOGY READINESS INDEX (TRI) NA CIDADE DE CATALÃO, GO
}

\author{
THE TECHNOLOGY READINESS: A STUDY ON THE \\ IMPLEMENTATIONOF THE TECHNOLOGY READINESS INDEX \\ (TRI) IN THE CATALÃO CITY -GO
}

Recebido 26/09/2010

Aceito 24/09/2011

Tércio William Pereira Rocha ${ }^{1}$ e Solon Bevilacqua ${ }^{2}$

\section{RESUMO}

Este é um estudo descritivo e exploratório que objetivou identificar preliminarmente o nível de prontidão à tecnologia na cidade de Catalão, GO, a partir de uma amostra de 368 indivíduos. O estudo utilizou a técnica Technology Readiness Index (TRI), proposta por Parasuraman e Colby (2002). Após a realização das análises, verificou-se que, apesar de a estrutura da TRI ter apresentado alterações em relação ao estudo original, foi possível constatar a aplicabilidade da escala no contexto da cidade alvo, levantar o perfil da amostra investigada, bem como identificar o seu nível de prontidão à tecnologia, que apresentou características de consumidores "Pioneiros", além de levantar aspectos gerais para a realização de futuras pesquisas causais ou conclusivas. Index (TRI).

Palavras-chave: Comportamento do consumidor. Prontidão à tecnologia. Technology Readiness

\footnotetext{
1 Mestrando em Administração pela Universidade Federal de Goiás (UFG). E-mail: twprmozart@hotmail.com 2 Doutorando em Psicologia pela PUCGO, mestre em Administração pela UFG, professor do departamento de Administração. E-mail: solbev@gmail.com
} 
This is a descriptive and exploratory study which aimed at identifying preliminarily the level of technology readiness in Catalan-GO, from a sample of 368 individuals. The study used the Technology Readiness Index (TRI) technique, proposed by Parasuraman and Colby (2002). After analysis completion, it was found that, although the structure of TRI had been different from the original study, it was possible to verify the measure applicability in the context of the city, to draw the profile of the sample investigated, as well as to identify its technology readiness level, which had presented characteristics of "Pioneer" consumers, besides to raise general aspects for the realization of future causal or conclusive research.

Keywords: Consumer behavior. Technology readiness. Technology Readiness Index (TRI).

\section{INTRODUÇÃO}

As organizações que conhecem profundamente o seu mercado de atuação concentram seus esforços para supri-lo adequadamente, oferecendo uma extensa linha de produtos, efetivamente inovadores e diferenciados, voltados para públicos segmentados.

Nessa perspectiva, clarifica-se a importância do planejamento de marketing para o desenvolvimento das ações organizacionais, no sentido de identificar as necessidades do mercado, preocupando-se em entregar, ao fim, algo que represente valor para o cliente.

Entretanto, um dos grandes desafios das organizações reside na compreensão dos fatores que determinam o comportamento do consumidor, considerando as distintas reações dele para iguais estímulos.

A complexidade do tema torna-se mais saliente quando se verifica que o esforço de marketing, direcionado a produtos e serviços baseados em tecnologia, é conduzido para princípios tradicionais, que podem não ser tão eficazes para oferecer produtos de alta tecnologia quanto o foram para vender aqueles nem tão complexos.

A partir dessas reflexões, dá-se o desenvolvimento deste estudo. Para tanto, a investigação se fundamentará nos trabalhos desenvolvidos por Parasuraman (2000) e Parasuraman e Colby (2001), que desenvolveram o construto da Prontidão para Tecnologia (Technology Readiness - TR) - que diz respeito à predisposição dos indivíduos para consumir produtos e serviços tecnológicos -, e também a escala de mensuração para essa Prontidão para Tecnologia (Technology Readiness Index - TRI).

Serão investigadas também as contribuições apresentadas por Souza e Luce (2003), que aplicou e validou a TRI no contexto brasileiro, partindo dos pressupostos balizados no contexto americano pelos autores supracitados.

Após essas considerações, emerge o problema de pesquisa que orientará os caminhos deste estudo: qual a prontidão à tecnologia verificada na cidade de Catalão, Goiás, com base nos critérios da Technology Readiness Index (TRI)?

\section{ADOÇÃO DE PRODUTOS COM CONTEÚDO TECNOLÓGICO}

A difusão de inovação tem recebido atenção singular nas últimas décadas em função de sua ênfase de pesquisa estar centrada na investigação de novos produtos, conforme Schiffman e Kanuk (2000). Assim sendo, os teóricos da área do Comportamento do Consumidor, a partir dos 
estudos antropológicos e sociológicos, adotaram o modelo geral da difusão para explicar o processo da adoção de novos produtos.

\subsection{Conceituando inovação}

Para Rogers (2003), inovação é uma ideia, prática ou objeto que é percebido como novo por um indivíduo ou outra unidade de adoção, não importando se a ideia é nova ou não, e sim se o indivíduo a percebe como tal. Autores como Engel, Blackwell e Miniard (2000) e Gatignon e Robertson (1991) criticam a obra de Rogers (2003) no tocante às dificuldades de operacionalização da definição de inovação, uma vez que esta depende da percepção de cada indivíduo. Outras críticas são somadas, afinal inovação de produto ou produto novo são expressões questionáveis, conforme Schiffman e Kanuk (2000). Para contornar essa deficiência, os autores trazem abordagens que podem ser classificadas como definições de inovação orientadas para a empresa, para o produto, para o mercado e para o consumo.

Segundo as considerações de Rogers (2003), Engel, Blackwell e Miniard (2000), Gatignon e Robertson (1991) e Schiffman e Kanuk (2000), foi possível delimitar, na etapa exploratória deste estudo, o conceito de inovação para a amostra pesquisada: a novidade lançada é gerada por meio de pesquisas e desenvolvimento, que aumenta a eficiência do processo produtivo. Para parcela expressiva dos entrevistados, a inovação deve trazer impacto positivo nos resultados financeiros da entidade.

A partir desses conceitos preliminares, Mukherjee e Hoyer (2001) afirmam que a adição de novos atributos provavelmente aumenta a avaliação e venda de um determinado produto, em função de o consumidor interpretar esses atributos como benefícios adicionais fornecidos pelo fabricante. Esse processo de adoção seria composto por várias fases, iniciando com o conhecimento e findando na adoção ou rejeição da nova ideia. Segundo Rogers (2003), as pessoas não adotam uma inovação simultaneamente. A adoção ocorre em uma sequência linear, possibilitando uma classificação para esses adotantes. Cada categoria seria composta por indivíduos que compartilham um grau de inovação semelhante, o que torna conveniente a descrição dos membros pertencentes a um sistema social.

Com relação à adoção de produtos com forte apelo tecnológico, Parasuraman (2000) sustenta que, em tal situação, não se percebe um real benefício esperado para o consumidor. $O$ motivo para essa situação é decorrente do aumento de frustrações no trato com sistemas ou aparatos tecnológicos. No entendimento de Meuter et al. (2000), os maiores problemas estão relacionados ao autosserviço, quando os clientes interagem com máquinas e equipamentos. Parasuraman (2000) afirma que tal aspecto cria a necessidade de amplo estudo, pois ainda se consideram incipientes as pesquisas acadêmicas referentes à prontidão das pessoas para o uso de tais sistemas.

Na mesma linha de pensamento, Mowen e Minor (2003) corroboram tal percepção do autor, mas esclarecem que as mudanças de ordem tecnológica podem mudar significativamente o modo de vida dos consumidores, independente da percepção desses benefícios. Nessa perspectiva, Mick e Fournier (1998) afirmam que nem sempre essas mudanças agregam sentimentos positivos de controle, liberdade, novidade, competência, eficiência, satisfação, associação e engajamento, pois podem também provocar sentimentos de caos, escravização, obsolescência, incompetência, ineficiência, insatisfação, isolamento e desengajamento.

Os conceitos de Cowles e Crosby (1990) são complementares a partir de outras pesquisas que procuraram investigar as reações do consumidor diante da tecnologia e apontaram 
paradoxos subjacentes à adoção de tecnologia. Apesar desses esforços mais recentes, existem ainda poucas investigações sobre a prontidão dos consumidores para usar produtos e serviços baseados em tecnologia.

Nesse sentido, a mudança tecnológica para o consumidor nem sempre tem caráter positivo, podendo ser paradoxal. Swinyard e Ghee (1987) e Thornton e White (2001) citam os trabalhos em que é destacada a importância de características atitudinais na diferenciação entre usuários e não usuários de produtos e serviços financeiros. Por exemplo, aqueles clientes com atitudes favoráveis à tecnologia, a computadores e à conveniência costumam ser mais independentes do atendimento efetuado por pessoas, preferindo os canais eletrônicos de autoatendimento, como caixas automáticos e Internet banking. Por outro lado, os clientes que não gostam de tecnologia e que não se sentem à vontade com serviços de autoatendimento tendem a limitar ao mínimo possível a interação com os referidos equipamentos.

Para mensurar a prontidão para tecnologia, tem-se um número expressivo de métodos, mas, neste estudo especificamente, utilizar-se-ão unicamente o método proposto por Parasuraman e Colby (2001) e o de Davis (1989), por estarem mais próximos do objeto deste estudo, cujas técnicas são evidenciadas pelo crescente emprego em publicações recentes, recebendo destaque: Kleijnen, Lee e Wetzels (2009); Kulviwat, Bruner e Shuridah (2009); Constantiou, (2009); Seneler, Basoglu e Daim (2009); Van Rijnsoever et al. (2009); Cantor, Corsi e Grimm (2008).

\section{TECHNOLOGY READINESS INDEX (TRI)}

A Technology Readiness Index (TRI) é fruto de extensa investigação de Parasuraman professor de marketing da University of Miami, também conhecido pela coautoria da escala de mensuração de qualidade percebida em serviços (SERVQUAL) - e Colby - presidente da Rockbridge Associates, empresa de consultoria e pesquisa especializada em temas relacionados à tecnologia (SOUZA; LUCE, 2003).

De acordo com Parasuraman e Colby (2001), a Technology Readiness Index é formada por 4 dimensões com 36 indicadores da prontidão para tecnologia, conforme apresentado na tabela 1.

Dessa forma, segundo os autores, a combinação dessas dimensões irá resultar na formação de cinco tipos de consumidores, que são:

- exploradores: apresentam altos índices de prontidão para tecnologia, com altos escores nas dimensões condutoras da adoção, otimismo e inovatividade; e baixos escores nas dimensões inibidoras, desconforto e insegurança;

- pioneiros: dividem com os exploradores altos níveis de otimismo e inovatividade, mas, ao mesmo tempo, apresentam níveis também altos de desconforto e insegurança;

- céticos: revelam escores baixos em todas as dimensões;

- paranoicos: apresentam altos níveis de otimismo, mas revelam níveis igualmente altos nas dimensões inibidoras da adoção. Este grupo apresenta, ainda, baixo grau de inovatividade;

- retardatários: representam o oposto dos exploradores, pois exibem baixos escores nas dimensões condutoras da adoção e altos escores nas dimensões inibidoras.

Essas características percebidas quando da inovação são formadas por cinco atributos primários, os quais afetam a taxa de adoção. Seriam eles: (1) vantagem relativa, (2) compatibilidade, (3) complexidade, (4) experimentabilidade e (5) observabilidade. 
Com relação a esses atributos, Souza e Luce (2005) argumentam que cada segmento, além de possuir padrões de crenças e sentimentos divergentes a respeito da tecnologia, também pode apresentar diferenças demográficas e psicográficas. Entretanto, esses atributos são suficientemente distintos para auxiliar as organizações na busca do melhor gerenciamento da relação cliente-tecnologia, bem como na consequente customização das estratégias voltadas à abordagem do tema. Desse modo, indivíduos considerados "paranoicos", por exemplo, necessitam de garantias técnicas de funcionamento e segurança, para adquirir um determinado produto/serviço, enquanto um indivíduo pertencente ao grupo dos "céticos" precisa ser convencido dos aspectos positivos do uso da tecnologia.

Posto isso com relação à tecnologia, Souza e Luce (2005) afirmam que a utilização do TRI se caracteriza como um instrumento de mensuração das atitudes e crenças dos indivíduos extremamente útil para predizer comportamentos de adoção, identificar tipos distintos de indivíduos e prever, de maneira mais acurada, comportamentos futuros de clientes e produtos.

\section{METODOLOGIA}

Este estudo foi realizado em duas etapas, conforme o processo utilizado por Souza e Luce (2003), sendo estas divididas em: etapa exploratória e etapa descritiva. Na primeira, realizou-se um minucioso levantamento teórico acerca dos temas pertinentes ao estudo da prontidão à tecnologia, como também a elaboração do instrumento de coleta de dados - a partir da versão aplicada e validada no contexto brasileiro por Souza e Luce (2003) - e o pré-teste desse instrumento.

A pesquisa pode ser classificada, em função de sua natureza, como básica; do ponto de vista da abordagem do problema, como quali e quantitativa; do ponto de vista de seus objetivos, como exploratória e descritiva; e do ponto de vista dos procedimentos técnicos, como experimental.

Quanto aos objetivos, este estudo foi realizado mediante o desenvolvimento de uma pesquisa exploratório-descritiva, caracterizando-se pela sua natureza quantitativa.

\subsection{Definição do universo da pesquisa e seleção da amostra}

Partindo do pressuposto de que este estudo pretendia aplicar uma escala para aferir a prontidão à tecnologia do consumidor, o universo da pesquisa do estudo abrangeu homens e mulheres acima de 18 anos, assim como no estudo original de Parasuraman e Colby (2001) e no estudo aplicado no contexto brasileiro por Souza e Luce (2003). As razões para tanto residem na consideração de que essa parcela da população detém, de fato, a autonomia e discernimento para adotar produtos e serviços.

A definição do tamanho da amostra obedeceu aos pressupostos básicos apresentados por Hair Jr. et al. (2005) para a adequada realização da Análise Fatorial (técnica de análise de dados utilizada, conforme será detalhado no subitem 4.2), isto é, entre 10 a 20 casos por variável. Assim, o tamanho da amostra foi definido em 360 casos, ou 36 variáveis x 10 casos. Entretanto, devido à identificação de 16 perfis, mediante a combinação das propriedades sexo, faixa etária e grau de instrução, o tamanho da amostra final foi estendido em 368 casos (23 pessoas por perfil). 
Inicialmente, a combinação das propriedades também contaria com a propriedade renda familiar mensal. Entretanto, a combinação elevaria consideravelmente a quantidade de perfis da amostra a ser investigada, o que poderia comprometer a realização do trabalho, pelas limitações de tempo. Dessa forma, optou-se por retirar essa propriedade da composição dos perfis, mantendo-a somente no questionário, para auxiliar na caracterização da amostra.

A adoção dessas variáveis busca avalizar a relevância da amostra investigada, conforme os objetivos deste estudo. Diante disso, considerou-se que as variáveis escolhidas contemplam características consonantes à proposta apresentada, qual seja, analisar a predisposição para adoção de produtos e serviços tecnológicos no contexto de Catalão. Quanto à identificação dos perfis, a finalidade recai na tentativa de dispersar eventuais vieses na escolha dos integrantes da amostra.

Os dados foram coletados entre os dias 17 de abril e 28 de maio de 2010, por meio de entrevistas diretas a indivíduos pertencentes aos 16 perfis delimitados, na cidade de Catalão, GO.

\subsection{Elaboração do instrumento e técnica de análise}

A versão da escala TRI adotada para este estudo é semelhante à utilizada por Souza e Luce (2003) e validada para o contexto brasileiro. A composição do instrumento de coleta foi dividida em duas seções:

1. Escala TRI: 36 afirmações sobre a tecnologia, abrangendo aspectos relativos às dimensões Otimismo, Inovatividade, Desconforto e Insegurança com produtos e serviços baseados em tecnologia. Os entrevistados atribuíram graus de concordância de 1 a 5 às afirmações, sendo 1 referente a "Discordo Totalmente" e 5 referente a "Concordo Totalmente";

2. Caracterização do entrevistado: foram inseridas questões pertinentes às características dos respondentes, como faixa etária, grau de instrução, renda familiar e sexo.

Para este estudo, realizaram-se 20 entrevistas com indivíduos integrantes da amostra definida. Os entrevistados demonstraram fácil compreensão dos itens da escala TRI, itens que não sofreram nenhuma alteração, a não ser pequenos ajustes na forma escrita de algumas variáveis, sem afetar, contudo, seu significado, além de mudanças em algumas questões elaboradas para identificação do perfil dos entrevistados.

\section{ANÁLISE DOS RESULTADOS}

A primeira parte apresenta o perfil dos entrevistados da amostra investigada, de acordo com as variáveis sexo, faixa etária, grau de instrução e renda familiar mensal.

Na segunda parte, são expostos os resultados concernentes à avaliação da aplicabilidade da Technology Readiness Index na cidade de Catalão, GO, a partir do exame da validade (de conteúdo e de construto) do instrumento, sendo esta verificada mediante o emprego da Análise Fatorial das dimensões encontradas. 


\subsection{Perfil da amostra}

O estudo apontou algumas particularidades da amostra, apresentadas a seguir:

- observou-se a predominância do segmento com "Ensino Médio Completo" (45\%), seguido pelos segmentos "Ensino Superior Completo" (29\%) e "Ensino Superior Incompleto" (21\%);

- apenas 5\% da amostra é composta pelo segmento "Ensino Médio Incompleto";

- no que se refere à distribuição dos respondentes por renda familiar mensal, a amostra se constitui de $45 \%$ de pessoas com renda entre $R \$ 1.020,00$ e $R \$ 3.060,00 ; 35 \%$ entre $\mathrm{R} \$ 3.060,00$ e $\mathrm{R} \$ 7.650,00 ; 10 \%$ com renda até $\mathrm{R} \$ 1.020,00 ; 8 \%$ entre $\mathrm{R} \$ 7.650,00 \mathrm{e}$ $\mathrm{R} \$ 15.300,00$; e $2 \%$ com renda acima de $\mathrm{R} \$ 15.300,00$.

\subsection{Avaliação da Technology Readiness Index}

A verificação da aplicabilidade da Technology Readiness Index (TRI) foi realizada mediante a utilização do software Systat 12. Para a identificação da estabilidade e a consequente validação da Análise Fatorial (AF), Hair Jr. et al. (2005) sugerem dividir a amostra em duas partes e rodar a AF para ambas as amostras. Neste caso específico, não se verificou anormalidade, obtendo-se, por conseguinte, a estabilidade para o modelo.

A presença de multicolinearidade normalmente prejudica a análise de um modelo de $A F$, por reduzir o valor do coeficiente de determinação, podendo ainda eliminar o efeito sobre as variáveis independentes. Hair Jr. et al. (2005) sugerem verificar os coeficientes de correlação de Spearman e identificar se as correlações entre as variáveis independentes do modelo variam entre 0,000 e 0,70. O emprego de tal teste sugeriu ausência de multicolinearidade entre as variáveis.

Objetivando avaliar a precisão da escala adotada, empregou-se o coeficiente de confiabilidade, que avalia a consistência integral da escala, o AlphadeCronbach, medida indicada para avaliar a correlação entre os itens de um instrumento. Quanto maior a correlação entre os itens, maior será o valor de á. Dessa forma, o teste também é conhecido como consistência interna do teste, e seus valores variam entre um e infinito negativo (embora apenas valores positivos façam sentido). Como regra geral, é posto que um bom valor do á seria 0,70 ou superior (HAIR JR. et al., 2005). Nos procedimentos, encontrou-se o valor de 0,86, tido como excelente. Outro teste empregado, e considerado satisfatório, foi o teste de esfericidade de Bartlett (2001), que busca demonstrar a probabilidade de não haver correlações entre as variáveis da matriz de correlação (hipótese nula). Este teste acusou um valor elevado, acompanhado de uma significância de 0,05, que indica o emprego da Análise Fatorial, conforme os autores supracitados.

Finalmente, foi aplicado o teste final para identificação da correlação entre os pares de variáveis. Para valores acima de 0,60 é recomendável o uso da Análise Fatorial, de acordo com Aaker, Kumar e Day (2001). Essa estatística compara o valor das correlações observadas com os valores das correlações parciais. Hair Jr. et al. (2005) e Malhotra (2001) indicam valores próximos de 0,50 para a estatística de Kaiser-Meyer-Olkin (KMO).

A partir da análise da estrutura fatorial, foram constatadas pontuais diferenças em relação ao estudo original de Parasuraman (2000), as quais serão apresentadas a seguir:

1. Entusiasmo (itens 1 a 11): neste estudo, a dimensão Otimismo apresentou mudanças relacionadas à variável 11, "Outras pessoas Ihe pedem conselhos sobre novas tecnologias", que evidenciou uma carga elevada para essa dimensão, diferindo do estudo original, em que essas cargas elevadas se davam na dimensão Inovatividade. Dessa forma, com a inserção da variável 11, optou-se por alterar a denominação da dimensão Otimismo, 
devido à característica contida no sentido da referida variável, que transcende o sentido inerente à dimensão. Isto porque outras pessoas geralmente pedem conselhos sobre tecnologia a entusiastas no assunto, que o dominem suficientemente, a ponto de serem vistos como referência de conhecimento sobre o tema em seus grupos de relacionamento. Com isso, a dimensão recebeu a denominação de Entusiasmo;

2. Inovatividade (itens 13 a 17): a estrutura encontrada não se comporta tal como no estudo original. A dimensão apresentou mudanças em relação às variáveis 11, que foi inserida na dimensão Entusiasmo, conforme relatado acima, e 12, "Parece que seus amigos estão aprendendo sobre as mais novas tecnologias mais do que você", que foi extraída por demonstrar comunalidade baixa, além de não contribuir para a melhoria da estrutura fatorial. Como evidenciado na tabela 1, as variáveis 16, "Você gosta do desafio de entender equipamentos de alta tecnologia", e 17, "Você tem menos problemas que outras pessoas para fazer a tecnologia trabalhar para você", também apresentaram cargas fatoriais consideráveis para a dimensão Entusiasmo. Entretanto, o conceito presente nas variáveis, além do valor das cargas apresentadas, fez com que se optasse em mantê-las na dimensão Inovatividade. A dimensão, contudo, manteve a mesma denominação, uma vez que as alterações realizadas não comprometeram a essência conceitual do fator;

3. Desconforto (itens 18, 19, 20, 21, 23, 25, 26 e 27): a dimensão apresentou mudanças em relação à variável 22, "Na compra de um produto ou serviço de alta tecnologia, você prefere o modelo básico a um modelo com muitas características adicionais", que, a exemplo da variável 12, também foi extraída, por evidenciar comunalidade baixa e por não contribuir para a melhoria da estrutura fatorial; e à variável 24, "Deveria haver cuidado ao substituir tarefas desempenhadas por pessoas pela tecnologia, pois novas tecnologias podem falhar", que apresentou uma carga fatorial elevada para a dimensão Insegurança, sendo, dessa forma, redistribuída para esta última dimensão. As variáveis 23, "É constrangedor quando você tem problemas com algum equipamento de alta tecnologia, enquanto outras pessoas estão olhando", 25, "Muitas das novas tecnologias apresentam riscos à saúde ou à segurança que não são descobertos até que as pessoas tenham utilizado a tecnologia", 26, "Novas tecnologias tornam muito fácil para o governo e as empresas poder espionar as pessoas", e 27, "As tecnologias sempre parecem falhar no pior momento possível", manifestaram comunalidades relativamente consideráveis para a dimensão Insegurança. Porém, assim como no caso das variáveis 16 e 17, adotou-se como critério o valor da carga e o conceito presente nas variáveis. No caso específico das variáveis 23 e 26, apesar de elas terem exibido cargas fatoriais inferiores a 0,40, decidiu-se pela manutenção das variáveis na estrutura, pela consideração de que sua permanência traria maior estabilidade à escala. No entanto, apesar das particularidades evidenciadas, não se revelou a necessidade de alterar a denominação da dimensão, pelas mesmas razões relatadas no fator Inovatividade;

4. Insegurança (itens 24, 28, 29, 30, 31, 32, 33, 34, 35 e 36): a dimensão diferiu do estudo de Parasuraman (2000) no que diz respeito à variável 24, que exibiu uma carga elevada para esse fator, destoando do estudo original, em que as cargas elevadas da variável apontavam para a dimensão Desconforto. Todavia, optou-se por manter a denominação original desse fator, haja vista que a variável 24, ao conceituar que novas tecnologias podem falhar, expressa uma postura de insegurança em relação à tecnologia. Após isso, baseando-se nas definições pré-estabelecidas por Parasuraman e Colby (2001),

a nova configuração das dimensões, de acordo com os resultados deste estudo, seria:

1. Entusiasmo: indica grande interesse pelo estudo e utilização de novas tecnologias, apresentando visões positivas sobre o tema e crenças de que essa dimensão propicia aos indivíduos maior controle, flexibilidade e eficiência em seu cotidiano; 
2. Inovatividade: representa uma tendência do indivíduo a ser pioneiro na adoção de tecnologia;

3. Desconforto: denota a percepção de falta de controle sobre a tecnologia e o sentimento de ser oprimido por ela;

4. Insegurança: denota desconfiança da tecnologia e ceticismo com relação às próprias habilidades em utilizá-la de forma apropriada.

Individualmente, a variável 32, "Qualquer transação realizada eletronicamente deveria ser confirmada posteriormente por escrito", apresenta a carga fatorial mais expressiva do modelo, sendo seguida pela variável 34 , "O contato humano é muito importante quando se faz negócios com uma empresa", ambas pertencentes à dimensão Insegurança. Por outro lado, a dimensão Desconforto apresenta as duas variáveis com menor carga fatorial do modelo (23 e 26, como já abordado).

Tabela 1 - Cargas fatoriais

\begin{tabular}{|c|c|c|c|c|}
\hline Variável & Inovatividade & Otimismo & Desconforto & Insegurança \\
\hline VAR_1 & 0,43 & 0,59 & 0,18 & 0,06 \\
\hline VAR_2 & 0,34 & 0,63 & $\mathrm{O}, \mathrm{O} 4$ & 0,24 \\
\hline VAR_3 & 0,26 & 0,43 & $(0,22)$ & $\mathrm{O}, 01$ \\
\hline VAR_4 & 0,50 & 0,60 & $\mathrm{O}, \mathrm{01}$ & 0,06 \\
\hline VAR_5 & 0,44 & 0,48 & 0,15 & $(\mathrm{O}, 08)$ \\
\hline VAR_6 & 0,40 & 0,62 & $(0,41)$ & 0,17 \\
\hline VAR_7 & 0,40 & 0,63 & $\mathrm{O}, 07$ & 0,35 \\
\hline VAR_8 & 0,44 & 0,69 & 0,25 & 0,33 \\
\hline VAR_9 & 0,45 & 0,64 & 0,19 & 0,20 \\
\hline VAR_10 & 0,38 & 0,59 & $(0,37)$ & $\mathrm{O}, \mathrm{O} 3$ \\
\hline VAR_ 11 & $\mathrm{O}, 33$ & 0,53 & O,01) & 0,12 \\
\hline VAR_12 & 0,22 & 0,21 & $(\mathrm{O}, \mathrm{O} 2)$ & 0,51 \\
\hline VAR_13 & 0,37 & $(0,48)$ & $(0,21)$ & 0,52 \\
\hline VAR_14 & 0,31 & $(0,47)$ & $(0,32)$ & 0,68 \\
\hline VAR_15 & 0,32 & $(0,46)$ & $(0,25)$ & 0,61 \\
\hline VAR_16 & 0,42 & 0,43 & $(0,47)$ & 0,50 \\
\hline VAR_17 & 0,39 & 0,41 & $(0,15)$ & 0,56 \\
\hline VAR_18 & 0,31 & 0,19 & 0,63 & $\mathrm{O}, 03$ \\
\hline VAR_19 & 0,40 & 0,21 & 0,67 & 0,09 \\
\hline VAR_2O & 0,40 & 0,31 & 0,67 & 0,07 \\
\hline VAR_21 & 0,33 & 0,28 & 0,68 & 0,24 \\
\hline VAR_22 & 0,24 & 0,15 & 0,21 & 0,37 \\
\hline VAR_23 & 0,32 & 0,20 & 0,39 & 0,06 \\
\hline VAR_24 & 0,48 & 0,25 & $(0,03)$ & $(0,17)$ \\
\hline VAR_25 & $\mathrm{O}, 42$ & $\mathrm{O}, 53$ & 0,42 & $(\mathrm{O}, \mathrm{O} 4)$ \\
\hline VAR_26 & 0,32 & 0,14 & 0,33 & $(0,02)$ \\
\hline VAR_27 & 0,40 & 0,34 & 0,43 & $(0,05)$ \\
\hline VAR_28 & 0,59 & 0,41 & 0,13 & 0,09 \\
\hline VAR_29 & 0,58 & 0,41 & 0,13 & 0,09 \\
\hline VAR_30 & 0,64 & 0,45 & 0,07 & $(0,00)$ \\
\hline VAR_31 & 0,64 & 0,43 & 0,13 & $\mathrm{O}, \mathrm{O} 2$ \\
\hline VAR_32 & 0,74 & 0,40 & 0,26 & $(0,16)$ \\
\hline VAR_33 & 0,67 & 0,50 & 0,20 & 0,31 \\
\hline VAR_34 & 0,70 & 0,54 & 0,27 & 0,27 \\
\hline VAR_35 & 0,67 & 0,23 & 0,33 & $(0,20)$ \\
\hline VAR_36 & 0,47 & 0,24 & 0,22 & $(0,09)$ \\
\hline
\end{tabular}

Fonte: coleta de dados. 
Todavia, de um modo geral, vale dizer que, a partir da observação dos resultados evidenciados no modelo, todas as dimensões exibiram cargas fatoriais consideravelmente satisfatórias (tanto as dimensões inibidoras - Desconforto e Insegurança -, quanto as dimensões condutoras - Entusiasmo e Inovatividade), o que, de acordo com as diferentes crenças dos segmentos de adoção de tecnologia, categorizam a amostra investigada como "Pioneiros". Isto é, esses indivíduos apresentam uma alta predisposição à adoção de produtos e serviços tecnológicos, mas também são mais práticos ao considerar as dificuldades e os perigos incorridos na tecnologia.

\section{CONSIDERAÇÕES FINAIS}

Este estudo apresentou como objetivo principal realizar um estudo preliminar sobre a prontidão à tecnologia na cidade de Catalão, mediante a aplicação da Technology Readiness Index (TRI) - instrumento adotado para mensurar a predisposição à adoção de produtos e serviços tecnológicos no contexto investigado, desenvolvido a partir dos estudos de Parasuraman (2000) e Parasuraman e Colby (2001).

Além desse objetivo, foram elencados quatro objetivos secundários, quer sejam: levantar o perfil dos entrevistados, com relação a variáveis como sexo, faixa etária, grau de instrução e renda familiar; verificar a aplicabilidade do constructo da Technology Readiness Index no contexto de Catalão; identificar o nível de prontidão da amostra estudada para consumir produtos e serviços tecnológicos; e levantar aspectos relacionados à prontidão para adoção de tecnologia na cidade de Catalão, de modo a fornecer subsídios para a realização de um estudo causal ou conclusivo.

Para tanto, inicialmente foi definida a amostra a ser analisada, que contou com um total de 368 pessoas, constituídas por homens e mulheres acima de 18 anos, que foram identificadas através de 16 perfis gerados pela combinação das propriedades sexo, faixa etária e grau de instrução. Para a caracterização da amostra, foi utilizada ainda a propriedade renda familiar mensal.

A partir disso, em relação ao primeiro objetivo específico, a distribuição da amostra ficou caracterizada da seguinte forma: $50 \%$ de homens e 50\% de mulheres, na distribuição por sexo; divisão igualitária entre as quatro faixas etárias ( $25 \%$ para cada porção); $50 \%$ das amostras caracterizadas por "Ensino Médio" (45\% completo e 5\% incompleto) e 50\% por "Ensino Superior" (29\% completo e 21\% incompleto), em relação ao grau de instrução; e $45 \%$ das pessoas com renda entre $R \$ 1.020,00$ e $R \$ 3.060,00,35 \%$ entre $R \$ 3.060,00$ e $R \$ 7.650,00,10 \%$ com renda até $R \$ 1.020,00$, 8\% entre $R \$ 7.650,00$ e $R \$ 15.300,00$ e $2 \%$ com renda acima de $R \$$ $15.300,00$.

Após isso, no que se refere ao segundo objetivo, constatou-se a aplicabilidade do modelo, mediante: a verificação dos coeficientes de correlação de Spearman, que indicou a ausência de multicolinearidade entre as variáveis; a aplicação do coeficiente de confiabilidade AlphadeCronbach, obtendo um resultado de 0,86, consideravelmente alto, segundo Hair Jr. et al. (2005); o emprego do teste de esfericidade de Bartlett (2001), que exibiu um valor elevado $(374,964)$, com uma significância de 0,05, indicando a utilização da Análise Fatorial; e, também, por meio da aplicação da estatística de Kaiser-Meyer-Olkin, para identificação da correlação entre os pares de variáveis, alcançando um valor de 0,577.

A análise do conjunto de dados identificou que não houve confirmação da estrutura de dimensões estabelecida por Parasuraman (2000), apresentando variações significativas em todos os fatores. Dentre essas mudanças, salienta-se a alteração da denominação da dimensão 
Otimismo para Entusiasmo, devido à inserção da variável 11, "Outras pessoas lhe pedem conseIhos sobre novas tecnologias", que modificou a essência conceitual da dimensão, motivando, assim, a alteração. Verificou-se ainda a necessidade de se extrair as variáveis 12 e 22, com o intuito de aprimorar a estrutura interna da escala.

Quanto ao terceiro objetivo, observou-se que a característica geral dos respondentes apresenta elevados índices de entusiasmo e inovatividade com relação à adoção de produtos e serviços tecnológicos. Mas, por outro lado, os respondentes também demonstram alto grau de desconforto e insegurança, caracterizando-os como consumidores "Pioneiros". Isto indica que a amostra investigada possui alta prontidão à tecnologia, sendo os consumidores, no entanto, mais práticos ao também considerar as dificuldades e os perigos incorridos em sua adoção.

Estabelecidas essas considerações, torna-se necessário salientar que esses resultados e conclusões decorrem de um estudo assinalado pelas limitações impostas aos trabalhos de amostragem não probabilista, não podendo, portanto, ser generalizados. Entretanto, como o quarto objetivo específico consistia em levantar aspectos relacionados à escala TRI que subsidiem a realização de um estudo causal ou conclusivo, pelo fato de este estudo ser moldado segundo objetivos exploratório-descritivos, esses resultados e constatações poderão ser utilizados para futuros estudos causais ou conclusivos.

Diante disso, como sugestão para pesquisas futuras, ressalta-se a importância de serem realizados estudos diretamente relacionados aos resultados da avaliação da estrutura da escala TRI apresentada neste trabalho, sobretudo no que se refere à estrutura de suas dimensões.

Como sugere Souza e Luce (2003), uma importante contribuição viria de estudos desenvolvidos a partir de uma abordagem longitudinal, com o objetivo de revelar possíveis transformações nos níveis de prontidão para tecnologia ao longo do tempo, e também no que diz respeito à estabilidade do construto. $\mathrm{O}$ autor apresenta ainda as possibilidades de se investigar os antecedentes da prontidão para tecnologia (características demográficas ou psicográficas, por exemplo), bem como suas consequências, revelando, dessa forma, como campo de estudo a ser explorado, a análise do papel da satisfação dos consumidores com produtos e serviços tecnológicos.

\section{REFERÊNCIAS BIBLIOGRÁFICAS}

\author{
AAKER, D. A.; KUMAR, V.; DAY, G. S. Pesquisa \\ de marketing. São Paulo: Atlas, 2001.
}

BARTLETT, M. S. A note on multiplying factors for various chi-squared approximations. Journal of the Royal Statistical Society, Series B 16, p. 296-298, 2001.

CANTOR, D.; CORSI, T.; GRIMM, C. Determinants of motor carrier safety technology adoption. Transportation Research Part E: Logistics and Transportation Review, v. 44, n. 5, p. 932947, Sept. 2008.
CONSTANTIOU, J. Consumer behaviour in the mobile telecommunications' market: the individual's adoption decision of innovative services. Telematics and Informatics, v. 26, n. 3, p. 270-281, Aug. 2009.

COWLES, D.; CROSBY, L. A. Consumer acceptance of interactive media in service marketing encounters. The service Industries Journal, v. 10, n. 3, p. 521, 1990.

DAVIS, F. D. Perceived usefulness, perceived ease of use, and user acceptance of computer technology. MIS Quarterly, v. 13, n. 3, p. 319340, 1989.

ENGEL, J. F.; BLACKWELL, R. D.; MINIARD, P. W. Comportamento do consumidor. 8. ed. Rio de Janeiro: JC, 2000. 
GATIGNON, H.; ROBERTSON, T. S. Innovative decision processes. In: ROBERTSON, T. S.; KASSARJIAN, H. H. Handbook of consumer behavior. Englewood Cliffs: Prentice Hall, 1991. p 316-348.

HAIR JR., J. F. et al. Análise multivariada de dados. 5. ed. Porto Alegre: Bookman, 2005.

KLEIJNEN, M.; LEE, M.; WETZELS, M. An exploration of consumer resistance to innovation and its antecedents. Journal of Economic Psychology, v. 30, n. 3, p. 344-357, June 2009.

KULVIWAT, S.; BRUNER, G.; SHURIDAH, O. The role of social influence on adoption of high tech innovations: the moderating effect of public/private consumption. Journal of Business Research, v. 62, n. 7, p. 706-712, July 2009.

MALHOTRA, N. Pesquisa de marketing. 3. ed. Porto Alegre: Bookman, 2001.

MEUTER, M. L. et al. Self-service technologies: understanding customer satisfaction with technology-based service encounters. Journal of Marketing, v. 64, n. 3, p. 50-65, July 2000.

MICK, D. G.; FOURNIER, S. Paradoxes of technology: consumer cognizance, emotions, and coping strategies. Journal of Consumer Research, v. 25, p. 123-143, Sept. 1998.

MOWEN, J. C.; MINOR, M. S. Comportamento do consumidor. Tradução de Vera Jordan. 1. ed. São Paulo: Prentice Hall, 2003.

MUKHERJEE, A.; HOYER, W. D. The effect of novel attributes on product evaluation. Journal of Consumer Research, v. 28, p. 462-472, Dec. 2001.

PARASURAMAN, A. Technology Readiness Index (TRI): a multiple-item scale to measure readiness to embrace new technologies. Journalof Service Research, v. 2, n. 4, p. 307320, May 2000.

PARASURAMAN, A.; COLBY, C. L. Marketing para produtos inovadores: como e por que seus clientes adotam tecnologia. Porto Alegre: Bookman, 2002.
PARASURAMAN, A.; COLBY, C. Technoready marketing: how and why your customers adopt technology. New York: Free Press, 2001.

ROGERS, E. M. Diffusion of innovations. 5. ed. New York: Free Press, 2003.

SCHIFFMAN, L. G.; KANUK, L. L. Comportamento do consumidor. 6. ed. Rio de Janeiro: LTC, 2000.

SENELER, C.; BASOGLU, N.; DAIM, N. Interface feature prioritization for web services: case of online flight reservations.

Computers in HumanBehavior, v. 25, n. 4, p. 862-877, July 2009.

SOUZA, R. V. de; LUCE, F. B. Adoção de produtos e serviços baseados em

tecnologia: uma avaliação da aplicabilidade do Technology Readiness Index (TRI) no Brasil.

In: $27^{\circ}$ Encontro da ANPAD, 27., 2003, Atibaia. Anais... Atibaia: ANPAD, 2003.

SOUZA, R. V. de; LUCE, F. B. Avaliação da aplicabilidade do Technology Readiness Index (TRI) para a adoção de produtos e serviços baseados em tecnologia. Revista de Administração Contemporânea, Curitiba, v. 9, n. 3, jul./set. 2005. Disponível em: < http:// www.scielo.br/scielo.php?pid=S1415$65552005000300007 \&$ script $=$ sci_arttext $>$. Acesso em: 3 mar. 2010.

SWINYARD, W.; GHEE, L. Adoption patterns of new banking technology in Southeast Asia. International Journal of Bank Marketing, v. 5 , n. 4, p. 35-48, 1987.

THORNTON, J.; WHITE, L. Customer orientation and usage of financial distribution channels. The Journal of Services Marketing, v. 15, n. 3, p. 168-187, 2001.

VAN RIJNSOEVER, F. et al. Interdependent technology attributes and the diffusion of consumer electronics. Telematics and Informatics, v. 26, n. 4, p. 410-420, Nov. 2009. 